\title{
Die Bedeutung des demografischen Wandels für das österreichische Hochwasserrisikomanagement
}

\author{
Ralf Nordbeck • Christoph Clar · Sven Fuchs · Lukas Löschner · Maria Papathoma-Köhle · Thomas Thaler • \\ Tatjana Fischer
}

Online publiziert: 3. März 2020

(C) Der/die Autor(en) 2020

\begin{abstract}
Zusammenfassung Der demografische Wandel zählt zu einer der bedeutendsten Herausforderungen unserer Zeit. Demografische Veränderungen vollziehen sich auf unterschiedliche Weise und mit unterschiedlicher Dynamik: Alterung, Bevölkerungsrückgang, Veränderung der Haushaltsstrukturen und Zuwanderung. Wenngleich die steigende Lebenserwartung und die anhaltend niedrigen Geburtenraten langfristig zu einem wachsenden Anteil an älteren Menschen in der Gesamtbevölkerung führen, sind die unterschiedlichen Teilräume Stadt und Land in sehr unterschiedlichem Ausmaß (bereits) vom Bevölkerungsrückgang betroffen. Aufgrund seiner quantitativen Dimension und der sicht- und spürbaren Auswirkungen vor allem auf kleinräumiger bzw. lokaler Ebene - zu nennen sind hierbei vor allem die Unter- bzw. Überauslastung von Infrastruktur, der bauliche Leerstand und die sich einengenden finanziellen Handlungsspielräume der Gemeinden - wird der demografische Wandel als Phänomen bereits öffentlich wahrgenommen. Obgleich
\end{abstract}

Dr. R. Nordbeck $(\bowtie) \cdot$ Dr. C. Clar Institut für Wald-, Umweltund Ressourcenpolitik, Universität für Bodenkultur Wien, Feistmantelstraße 4, 1180 Wien, Österreich

ralf.nordbeck@boku.ac.at

PD Dr. S. Fuchs .

Dr. M. Papathoma-Köhle •

MMag. T. Thaler, Ph.D.

Institut für Alpine Naturgefahren, Universität für Bodenkultur Wien Peter-Jordan-Straße 82, 1190 Wien, Österreich

Mag. Dr. L. Löschner •

Mag. Dr. T. Fischer Institut für Raumplanung,

Umweltplanung und Bodenordnung, Universität für Bodenkultur Wien, Peter-Jordan-Straße 82, 1190 Wien, Österreich sich der demografische Wandel in vielerlei Hinsicht bereits manifestiert, wird ihm im Bereich des Hochwasserrisikomanagements sowohl in der wissenschaftlichen Auseinandersetzung als auch in der Politikgestaltung und -umsetzung bislang kaum Aufmerksamkeit geschenkt. Dieser Beitrag gibt einen Überblick über den Stand der Auseinandersetzung mit dem demografischen Wandel in Bezug auf Hochwasserrisikomanagement aus dem Blickwinkel von Expertinnen und Experten aus den Bereichen Naturgefahren- und Katastrophenmanagement sowie der Raumplanung. Die ExpertInnenumfrage zeigt, dass Zusammenhänge zwischen demografischem Wandel und dem Management von Hochwasserrisiken für die meisten Akteure in Österreich auf der Hand liegen und als sehr relevant für das zukünftige Hochwasserrisikomanagement eingestuft werden. Insbesondere die demografische Alterung und der Bevölkerungsrückgang, aber auch die anhaltenden Veränderungen in der Haushaltsstruktur (d.h. der zunehmende Anteil von Einpersonenhaushalten und Zweitwohnungen) werden in verschiedenen Phasen des Risikomanagementzyklus als zunehmend relevant eingeschätzt. Die Ergebnisse zeigen aber auch, dass in der gegenwärtigen Politikgestaltung und -umsetzung und hier insbesondere bei der Planung - diese langfristigen Entwicklungen kaum berücksichtigt werden.

\section{Schlüsselwörter}

Hochwasserrisikomanagement . demografischer Wandel · Alterung · Bevölkerungsrückgang

\section{The relevance of demographic change for Austrian flood risk management}

Abstract Demographic change is one of the most important challenges of our time. Demographic changes take place in different ways and with different dynamics: aging, population decline, changes in household structures and immigration. Although the increasing life expectancy and the persistently low birth rates lead to a growing proportion of older people in the total population in the long term, the different suburbs of urban and rural areas are (to a large extent) affected by population decline. Due to its quantitative dimension and the visible and noticeable effects, especially on a small-scale or local level-the underutilization or overutilization of infrastructure, the building vacancy rate and the narrowing financial scope of municipalities are to be mentioned here-the demographic change is already perceived as a phenomenon in public. Although the demographic change is already manifesting itself in many ways, it has so far received little attention in the area of flood risk management, both in the scientific debate and in policy-making and implementation. Using Austrian flood risk management as an example, this article provides an overview of the current state of engagement with demographic change in relation to flood risk management from the perspective of experts in the field of natural hazard and disaster management as well as spatial planning. The expert survey shows that correlations between demographic change and the management of flood risks are obvious for most actors in Austria and are classified as very relevant for future flood risk management. In particular, the demographic aging and population decline, but also the ongoing changes in the household structure (i.e. the increasing proportion of single-person households and second homes) are considered to be increasingly relevant in various phases of the risk management cycle. However, the results also show that these longterm developments are hardly taken into account in current policy-making, planning and implementation. 
Keywords Flood risk management . Demographic change · Aging Population decline

\section{Einleitung}

Der demografische Wandel in Österreich wird seit einigen Jahren vor allem im Bereich der Bereitstellung der öffentlichen Nahversorgung (Verkehrsmittel, Bildung, Gesundheit und Soziales) intensiv diskutiert und findet in der Raumentwicklung durchaus Berücksichtigung (Tappeiner et al. 2008; Bätzing 2015; ÖROK 2015). Für den Bereich Hochwasserrisikomanagement gilt das allerdings kaum. Vereinzelte Ansätze finden sich nur im Zusammenhang mit dem Bereich des Freiwilligenwesens bzw. der Rolle von Rettungsorganisationen im Katastrophenmanagement (Balas et al. 2015; BMASK 2015). Darüber hinaus werden die Zusammenhänge mit demografischen Entwicklungen und die Konsequenzen für das Hochwasserrisikomanagement in Österreich nur ungenügend thematisiert. In der wissenschaftlichen Literatur werden hauptsächlich die Auswirkungen des Bevölkerungswachstums diskutiert. Andere Ausprägungen des demografischen Wandels hingegen werden weitgehend ausgeblendet. Das erstaunt insofern, als davon auszugehen ist, dass sich Bevölkerungsrückgang, demografische Alterung sowie Änderungen von Haushaltsstrukturen infolge von geänderten Arbeits- und Lebensweisen in den nächsten Jahren und Jahrzehnten besonders auf kleinräumiger Ebene deutlich auswirken und in weiterer Folge erhebliche strukturelle Herausforderungen für die Bevölkerung vor Ort sowie die Qualität der Daseinsvorsorge mit sich bringen werden. Strukturelle Herausforderungen sind hierbei nicht alleine in Bezug auf technische Infrastrukturen (v. a. Verkehrswege und Trassen) und soziale Infrastrukturen (z. B. Ausbildungs- und Gesundheitseinrichtungen) evident, sondern auch in Bezug auf das Hochwasserrisikomanagement, sei es im Zusammenhang mit Prävention, dem Einsatz von Rettungsorganisationen oder beim Wiederaufbau (CoR 2016; Clar 2019).

Der Fokus wissenschaftlicher Diskussionen liegt diesbezüglich nach wie vor sehr stark auf der Aufrechterhaltung bzw. Wiederherstellung der öffentlichen Daseinsvorsorge in von Hochwasser betroffenen Gebieten und den möglichen Folgen für demografische Entwicklun- gen, aber nur sehr selten auf den neuen Herausforderungen für das Hochwasserrisikomanagement selbst. Obwohl demografische Merkmale in Untersuchungen zur Anfälligkeit bzw. Verletzbarkeit von Personen im Hinblick auf Hochwasserereignisse einfließen (Cutter et al. 2003; Fekete 2009a, 2009b, 2018; Birkmann et al. 2015; Karagiorgos et al. 2016), werden diese in der Planung und dem Management von Hochwasserrisiken in der Regel ausgeblendet. Es ist davon auszugehen, dass dies eine große Herausforderung für jene Gebiete im ländlichen Raum darstellt, die infolge von Wanderungsbewegungen in Richtung urbaner Zentren demografisch altern (Brown 2010).

Dem vorliegenden Artikel liegt die Annahme zugrunde, dass die Auswirkungen demografischer Entwicklungen auf Dauer nicht aus den Diskussionen über ein effektives Management von Hochwasserrisiken ausgeblendet werden können. Im Zuge des von der Österreichischen Akademie der Wissenschaften finanzierten Projekts „DemoHazAlps-Demographic change and hydrological hazards: flood risk management in Alpine areas facing population decline and demographic ageing" wird dieser Vermutung auf den Grund gegangen. $\mathrm{Zu}$ diesem Zweck wurde im Juni 2018 eine österreichweite OnlineBefragung von Expertinnen und Experten aus den Bereichen Naturgefahrenmanagement, Risikovorsorge und Raumplanung durchgeführt, die in einer Bestandsaufnahme des aktuellen Meinungsbildes von FachexpertInnen hinsichtlich der gegenwärtigen und zukünftigen Bedeutung des demografischen Wandels für das Hochwasserrisikomanagement in Österreich resultierte. Ziel der Befragung war es, einerseits die möglichen Auswirkungen zentraler Komponenten des demografischen Wandels - Alterung, Bevölkerungsrückgang, Pluralisierung sowie Veränderung von Haushaltsstrukturen - auf die verschiedenen Phasen des Hochwasserrisikomanagements in Österreich aus Sicht der relevanten Akteure zu erfassen, und andererseits potenzielle, dadurch ausgelöste Konflikte zu identifizieren.

Dieser Beitrag gibt einen Überblick über die wichtigsten Ergebnisse der Befragung und formuliert zentrale Schlussfolgerungen. Er ist wie folgt aufgebaut: In Abschn. 2 wird das methodische Vorgehen der Online-Umfrage vorgestellt. In Abschn. 3 wird der aktuelle Stand des demografischen
Wandels in Österreich kurz dargestellt und die daraus entstehenden neuen Herausforderungen für das Hochwasserrisikomanagement beleuchtet. In Abschn. 4. werden die wesentlichen Ergebnisse der Umfrage zusammengefasst, bevor in Abschn. 5. die zentralen Erkenntnisse herausgearbeitet werden und abschließend ein Ausblick auf die damit verbundenen zukünftigen Herausforderungen für das österreichische Hochwasserrisikomanagement gegeben wird.

\section{Material und Methodik}

Als Grundlage für die Online-Umfrage hat das interdisziplinäre Forschungsteam der Universität für Bodenkultur Wien (BOKU) im ersten Schritt einen Überblick über Akteure erstellt, die in Österreich für das Naturgefahrenund Katastrophenmanagement relevant sind. Die ExpertInnen aus diesen zentralen Institutionen und Organisationen sollten uns dabei helfen, die Relevanz des demografischen Wandels für die Gegenwart und Zukunft des Hochwasserrisikomanagements einzuschätzen. Das Ziel war, ein möglichst umfassendes Meinungsbild aus verschiedenen Bereichen des Naturgefahren- und Katastrophenmanagements $\mathrm{zu}$ erhalten. Aufbauend auf der Recherche wurden 108 Personen mittels persönlich adressierter E-Mail dazu eingeladen, an der Umfrage teilzunehmen. Eingeladen wurden ExpertInnen aus den einschlägigen Fachabteilungen (Schutzwasserwirtschaft, Wildbach- und Lawinenverbauung, Raumplanung) in den Ämtern der Landesregierungen und den zuständigen Bundesministerien, Vertreterinnen und Vertreter des Zivilund Katastrophenschutzes sowie von Blaulichtorganisationen (Feuerwehr, Rotes Kreuz, Arbeiter-Samariter-Bund, Johanniter, Malteser, Bergrettung) auf Bundes- und Landesebene.

In einem zweiten Schritt hat das interdisziplinäre Forschungsteam der BOKU einen Fragebogen erstellt und mithilfe des Umfragetools Limesurvey online gestellt. Die Teilnahme an der Umfrage war für die Dauer von 2 Wochen (18.06.-30.06.2018) möglich. Die Umfrage erfolgte auf freiwilliger Basis und anonym. Allerdings hatten die TeilnehmerInnen die Möglichkeit, am Ende der Umfrage ihre Kontaktdaten bekannt zu geben. Insgesamt beantworteten 53 Personen die Fragen, was einer Rücklaufquote von knapp $49 \%$ 
Tab. 1 Umfrageteilnehmerinnen und -teilnehmer aus den verschiedenen Fachbereichen

\begin{tabular}{|l|l|}
\hline Fachbereich & Anzahl \\
\hline (Schutz-)Wasserwirtschaft & 12 \\
\hline Wildbach- und Lawinenverbauung & 4 \\
\hline Raumplanung & 9 \\
\hline Zivil- und Katastrophenschutz & 7 \\
\hline Blaulichtorganisationen & 18 \\
\hline Sonstiges & 3 \\
\hline
\end{tabular}

entspricht (siehe Tab. 1). Die Rücklaufquote liegt damit weitaus höher als bei vergleichbaren Online-Umfragen. Dies spricht für ein großes Interesse der FachexpertInnen am Thema demografischer Wandel.

Der Online-Fragebogen bestand aus 21 Fragen (14 geschlossene sowie 7 offene Fragen), die den verschiedenen Phasen des Risikokreislaufs (Wiederaufbau, Instandsetzung, Einsatzmaßnahmen, Einsatzvorbereitung, Vorsorge, Prävention mithilfe technischer Maßnahmen sowie Prävention durch Planungsmaßnahmen) zugeordnet waren. Einem Fragenblock zu persönlichen Angaben der Befragten folgte ein Block zur Einschätzung der allgemeinen Relevanz des Themas „Demografischer Wandel“. Daran schlossen Fragen zur Relevanz des demografischen Wandels in den verschiedenen Bereichen des Risikokreislaufs an. Die Zustimmung $\mathrm{zu}$ bestimmten Aussagen oder Fragen wurde mithilfe einer dreiteiligen Ordinalskala $(1=$ hohe Relevanz; 2 =geringe Relevanz; 3 = kaum Relevanz) abgefragt. Darüber hinaus gab es die Möglichkeit $\mathrm{zu}$ eigenen textlichen Anmerkungen.

\section{Der demografische Wandel in \\ Österreich und mögliche Herausforderungen für das} Hochwasserrisikomanagement

Die Aufgabe der Demografie ist es, die aktuelle Zusammensetzung der Bevölkerung wie auch die strukturellen Veränderungen im Vergleich zu früheren Zeitpunkten zu beschreiben. Unter demografischem Wandel versteht man daher alle Veränderungen in der Struktur der Bevölkerung eines Landes über einen längeren Zeitraum. Als Kennzahlen zur Ermittlung der Bevölkerungsstruktur dienen dabei unter anderem die Altersstruktur, die Geburtenund Sterberate sowie die Migrationsstruktur. Für die europäischen Länder lassen sich generell zwei Entwicklun- gen feststellen: (i) ein Rückgang der Geburtenrate und (ii) ein Anstieg der Lebenserwartung. Wird in der Presse und den Medien von demografischem Wandel gesprochen, dann ist damit in der Regel diese langfristige, schleichende Entwicklung gemeint, die zu einem langsamen, jedoch stetigen Bevölkerungsrückgang führt. Zugleich erhöht sich in Verbindung mit der höheren Lebenserwartung das Durchschnittsalter der Bevölkerung. Dieser allgemeine Trend kann auch durch gegenläufige Tendenzen wie etwa eine positive Migrationsrate nur zum Teil aufgefangen werden.

Um den verschiedenen Facetten des demografischen Wandels gerecht $\mathrm{zu}$ werden, haben wir im Rahmen unserer Online-Umfrage vier verschiedene Ausprägungen des demografischen Wandels berücksichtigt:

1. Demografische Alterung: Übergang $\mathrm{zu}$ einer älteren Bevölkerungsstruktur aufgrund geringer Geburtenraten und hoher Lebenserwartung;

2. Bevölkerungsrückgang: Reduzierung der Einwohnerzahl einer Region aufgrund langfristiger demografischer Trends (zu geringe Geburtenrate oder Landflucht);

3. Veränderung von Haushaltsstrukturen: Abnahme der durchschnittlichen Haushaltsgröße, Zunahme von Zweitwohnsitzen und Trennung von Wohn- und Arbeitsort;

4. Migration und Pluralisierung: sich ändernde kulturelle und ethnische Zusammensetzung einer Bevölkerung durch Zuwanderung.

Betrachtet man den gegenwärtigen Stand des demografischen Wandels in Österreich aus dieser Perspektive - ergibt sich ein differenziertes Bild. Die Einwohnerzahl Österreichs erhöhte sich 2016 auf 8.772.865 EinwohnerInnen. Das Bevölkerungswachstum ging fast ausschließlich auf den Zuwanderungsüberschuss aus dem Ausland zurück. Nach den aktuellen Prognosen von Statistik Austria wird die Bevölkerung Österreichs auch in Zukunft anwachsen. Die Statistik Austria berechnet, dass die Einwohnerzahl im Jahresdurchschnitt 2025 bei anhaltender Entwicklung erstmals mehr als 9Mio. betragen wird. Gemäß mittlerer Variante der aktuellen Bevölkerungsvorausschätzung wird die Einwohnerzahl Österreichs bis 2030 auf 9,30 Mio. anwachsen (Statistik Austria 2017). Während der Anteil der unter 20-Jährigen an der Gesamtbevölkerung zwischen 2017 und 2030 relativ konstant bleiben wird (19,3\%), wird der Anteil der 20bis 64-Jährigen an der Gesamtbevölkerung sinken. Bis zum Jahr 2030 geht er von knapp 62 (2017) auf $57 \%$ zurück. Dies bedeutet auch einen Rückgang der Personen, die im erwerbsfähigen Alter stehen. Im Jahr 2017 sind 5,4 Mio. Personen im erwerbsfähigen Alter zwischen 20 und 65 Jahren gewesen. Bis 2030 wird sich die Zahl auf 5,3 Mio. verringern, da deutlich mehr Menschen ins Pensionsalter übertreten als Jugendliche aus der Ausbildung bzw. Zugewanderte hinzukommen (Báchora et al. 2016; Statistik Austria 2017). Die am stärksten wachsende Gruppe sind Menschen über 65 Jahre. Nach 2020 werden die BabyBoomer-Jahrgänge der späten 1950erund 1960er-Jahre in das Pensionsalter eintreten. Waren im Jahr 2013 noch 1,54 Mio. bzw. $18 \%$ der Bevölkerung 65 Jahre und älter, so werden im Jahr 2030 2,15 Mio. EinwohnerInnen über 65 Jahre alt sein (Báchora et al. 2016; Statistik Austria 2017).

Die demografische Entwicklung Österreichs ist darüber hinaus von einem starken Wachstum der großen Städte bei gleichzeitiger Bevölkerungsabnahme in peripheren ländlichen Regionen gekennzeichnet. Laut Zahlen der Statistik Austria findet unter Inländern eine Wanderbewegung von ländlichen in städtische Regionen statt. Das trifft nicht auf alle Altersgruppen $\mathrm{zu}$, aber besonders auf 18- bis 26-Jährige: Zwischen 2006 und 2015 haben pro Jahr etwa 6000 Menschen in diesem Alter ländliche Regionen verlassen, um in Ballungszentren zu ziehen. Auch in der Gruppe der 27- bis 39-Jährigen gibt es eine Landflucht - wobei hier Menschen eher in Speckgürtel ziehen. Die persönliche Verwirklichung suchen junge Menschen also in der Stadt, und zwar unabhängig davon, wo es die Jobs gibt. Deshalb fehlen in ländlichen Regionen des Westens, die oft auch Touristenmagnete sind, Arbeitskräfte (Szigetvari 2018).

Aus diesen demografischen Entwicklungen ergeben sich neue Herausforderungen für das Hochwasserrisikomanagement. In den Bereichen Vorbereitung und Vorsorge stellen sich Fragen nach dem Einfluss des demografischen Wandels auf die Bereitschaft zu langfristigen Investitionen in die Schutzinfrastruktur, der Rolle der Raumplanung bei der Bewältigung des Hochwasserrisikos in Regionen mit Bevölkerungsrückgang 
und den Auswirkungen des demografischen Wandels auf die organisatorischen Kapazitäten des Katastrophenschutzes. Mit Blick auf den Ereignisfall ist $\mathrm{zu}$ fragen, wie sich der demografische Wandel auf die Exposition und physische Anfälligkeit von Gebäuden in gefährdeten Gebieten und auf die soziale Vulnerabilität und die individuellen und gesellschaftlichen Kapazitäten zur Bewältigung eines Extremereignisses auswirkt. Im Bereich der Nachsorge stellt sich die Frage, inwiefern der demografische Wandel die individuellen und gesellschaftlichen Fähigkeiten beeinflusst, sich von Extremereignissen zu erholen.

\section{Die Auswirkungen des} demografischen Wandels auf das Hochwasserrisikomanagement aus Sicht der Akteure

\subsection{Relevanz des demografischen Wandels für das Hochwasserrisikomanagement in Österreich}

Die meisten Befragten gehen davon aus, dass die Folgen des demografischen Wandels in Zukunft Einfluss auf das Hochwasserrisikomanagement in Österreich haben werden. Bei der Frage nach der Relevanz des demografischen Wandels für das Hochwasserrisikomanagement in Österreich zeigt sich in den Antworten ein deutlicher Unterschied zwischen der gegenwärtigen und der zukünftigen Bedeutung dieses Themas. Gegenwärtig spielt der demografische Wandel für die meisten Akteure noch eine sehr geringe Rolle. Weniger als $10 \%$ der Befragten gaben an, dass der demografische Wandel schon jetzt eine starke Bedeutung für ihre Arbeit hat (siehe Abb. 1).

In Zukunft werden die demografischen Veränderungen nach Einschätzung der Befragten jedoch deutlich an Bedeutung gewinnen (siehe Abb. 2). Knapp die Hälfte ist der Meinung, dass die Bedeutung des demografischen Wandels für das Hochwasserrisikomanagement stark zunehmen wird. Betrachtet man die Antworten der Befragten in Bezug auf die Relevanz der verschiedenen Ausprägungen des demografischen Wandels, zeigen sich deutliche Unterschiede. Vor allem die Alterung der Bevölkerung wird als hochrelevant für das zukünftige Hochwasserrisikomanagement in Österreich eingeschätzt (51\%), dicht gefolgt vom
Bevölkerungsrückgang (47\%) und dem Wandel der Haushaltsstrukturen (42\%). Dem Thema Migration/Pluralisierung wird in diesem Zusammenhang eine deutlich geringere Bedeutung zugemessen (19\%).

\subsection{Die Bedeutung des demografischen Wandels im Kontext des} Risikokreislaufs

Im Bereich des Risikomanagements für Naturkatastrophen hat sich in Europa das Kreislaufmodell zum „Integralen Risikomanagement“ des Schweizer Bundesamtes für Bevölkerungsschutz durchgesetzt (BABS 2014). Dieser Kreislauf weist in seiner Grundstruktur drei Phasen auf: Vorbeugung (Prävention und Vorsorge), Bewältigung (Einsatzvorbereitung und Einsatz) und Regeneration (Instandhaltung und Wiederaufbau). Die befragten ExpertInnen bringen zahlreiche Herausforderungen, die mit demografischen Entwicklungen zusammenhängen, mit den verschiedenen Phasen des Risikokreislaufs in Verbindung. Die Antworten in unserer Umfrage zeigen, dass die langfristige demografische Alterung der Bevölkerung in Österreich nach Meinung der Befragten vor allem Auswirkungen auf

घar nicht $\square$ kaum $\square$ stark $\square$ kA

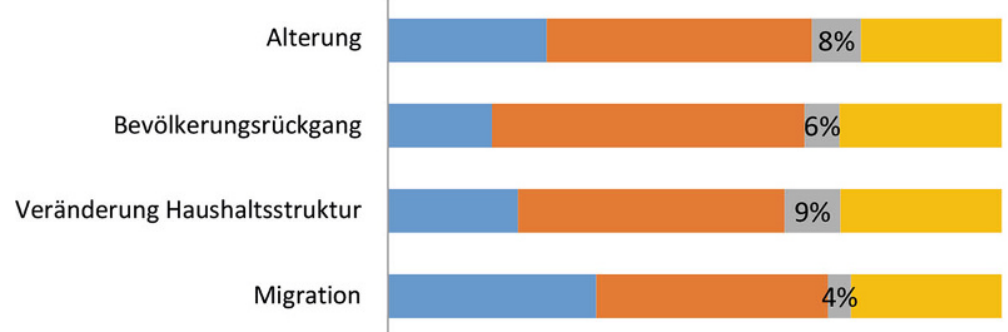

Abb. 1 Gegenwärtige Relevanz des demografischen Wandels für Hochwasserrisikomanagement

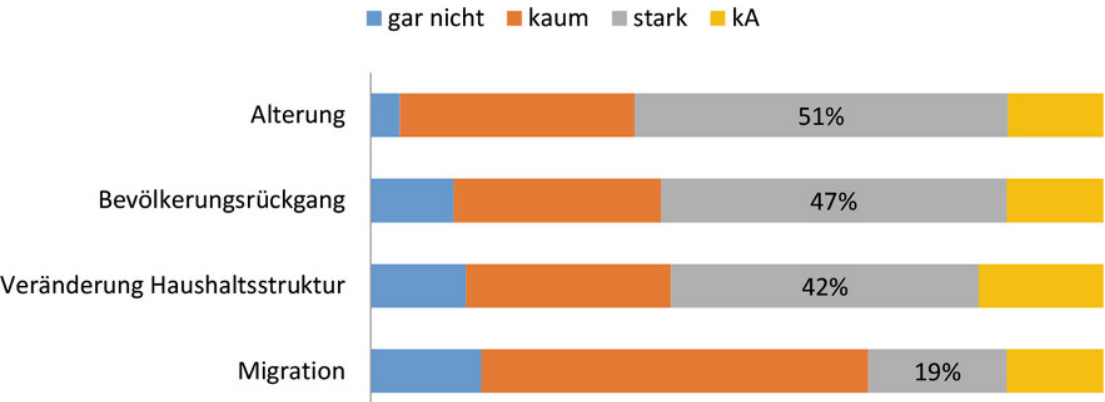

Abb. 2 Zukünftige Relevanz des demografischen Wandels für Hochwasserrisikomanagement 


\begin{tabular}{|c|c|c|c|c|}
\hline \multicolumn{5}{|c|}{ mar nicht $\square$ kaum $\square$ stark $\square$ keine Angabe } \\
\hline Wiederaufbau & $8 \%$ & & $42 \%$ & $21 \%$ \\
\hline Instandsetzung & $11 \%$ & $38 \%$ & $28 \%$ & $23 \%$ \\
\hline Einsatzmassnahmen & $8 \% \quad 15 \%$ & & $58 \%$ & $19 \%$ \\
\hline Einsatzvorbereitung & $4 \% \quad 17 \%$ & & $57 \%$ & $23 \%$ \\
\hline Vorsorge & $4 \% \quad 17 \%$ & & $60 \%$ & $19 \%$ \\
\hline Prävention (techn. Massnahmen) & $21 \%$ & $36 \%$ & $26 \%$ & $17 \%$ \\
\hline Prävention (plan. Massnahmen) & $9 \%$ & $32 \%$ & $40 \%$ & $19 \%$ \\
\hline
\end{tabular}

Abb. 3 Relevanz der Alterung in Bezug auf den Risikokreislauf

घar nicht $\quad$ kaum $=$ stark $=$ keine Angabe

\begin{tabular}{|c|c|c|c|c|}
\hline Wiederaufbau & $11 \%$ & $34 \%$ & $30 \%$ & $25 \%$ \\
\hline Instandsetzung & $13 \%$ & $34 \%$ & $26 \%$ & $26 \%$ \\
\hline Einsatzmassnahmen & $17 \%$ & $32 \%$ & $28 \%$ & $23 \%$ \\
\hline Einsatzvorbereitung & $15 \%$ & $28 \%$ & $32 \%$ & $25 \%$ \\
\hline Vorsorge & $6 \%$ & $28 \%$ & $45 \%$ & $21 \%$ \\
\hline Prävention (techn. Massnahmen) & $17 \%$ & $30 \%$ & $34 \%$ & $19 \%$ \\
\hline Prävention (plan. Massnahmen) & $6 \%$ & $26 \%$ & $45 \%$ & $23 \%$ \\
\hline
\end{tabular}

Abb. 4 Relevanz der Veränderung der Haushaltsstruktur in Bezug auf den Risikokreislauf

Aktuell bereits spürbare Folgen werden vor allem im Hinblick auf den Einsatz von Einsatzkräften während eines Hochwasserereignisses attestiert. Vor allem während eines Hochwasserereignisses sind ältere Personen besonders stark von der Unterstützung durch Blaulichtorganisationen abhängig. Gleichzeitig werden jedoch eben jene Organisationen durch Alterung und Bevölkerungsrückgang im ländlichen Raum vor Schwierigkeiten bei Rekrutierung und Bereitstellung einer ausreichenden Anzahl von aktiven Mitgliedern gestellt. Erschwerend kommt im Zusammenhang mit der Bereitstellung von einsatzfähigen Einsatzkräften hinzu, dass sehr viele erwerbstätige mögliche Relevanz für die Phase der Vorsorge hin. In Bezug auf eine demografisch alternde Bevölkerung wird meist die Frage diskutiert, wie einzelne Personen in Zukunft mit extremen Wetterereignissen umgehen bzw. wie sie sich auf solche Ereignisse vorbereiten (können). Die Befragten schreiben älteren Menschen eine besonders große Verletzlichkeit im Zusammenhang mit Hochwasserereignissen zu. Die hohe soziale Verletzbarkeit wird vor allem aus eingeschränkten körperlichen Fähigkeiten abgeleitet, die in eine eingeschränkte Mobilität und damit reduzierte Fähigkeit zur Selbsthilfe münden. Das wiederum wird durch die Tatsache erschwert, dass die Bevölkerung in Österreich insgesamt über ein international vergleichsweise geringes Wissen über und Interesse am Thema Naturgefahren verfügt und darüber hinaus häufig über eingeschränkte Möglichkeiten verfügt, sich individuell auf Hochwasserereignisse vorbereiten $\mathrm{zu}$ können. Die Sorglosigkeit in Bezug auf mögliche Hochwasserereignisse sowie die "Verleugnung“ der damit verbundenen Schäden führt häufig dazu, dass die Bereitschaft der Bevölkerung, konkrete Eigenvorsorgemaßnahmen zu setzen, vergleichsweise gering ist.

Neben der Alterung und dem Bevölkerungsrückgang ist vor allem die Veränderung der Haushaltsstrukturen von zentraler Bedeutung. Dies äußert sich in einer Verkleinerung der Haushaltsgrößen und einem wachsenden Anteil von Ein-Personen-Haushalten, die das Potenzial an innerfamiliärer Selbsthilfe während des Wiederaufbaus deutlich einschränken. Daraus lässt sich schließen, dass Blaulichtorganisationen und der öffentlichen Hand bei der Organisation und Koordination des Wiederaufbaus in Zukunft wahrscheinlich eine stärkere Bedeutung zukommen wird. Die Ergebnisse zeigen außerdem, dass infolge der Veränderung der Haushaltsstrukturen auch erhebliche Konsequenzen für die Vorsorge erwartet werden (siehe Abb. 4).

Gleichzeitig wird sich für Gemeinden, die von demografischer Alterung und Bevölkerungsrückgang betroffen sind, vermehrt die Frage stellen, ob technische Schutzmaßnahmen in $\mathrm{Zu}$ kunft überhaupt noch errichtet bzw. instandgehalten werden können. Beides wird von der Finanzkraft der Kommunen abhängen, die wiederum eng an die Anzahl und Entwicklung der Hauptwohnsitzbevölkerung geknüpft 
gar nicht $\quad$ kaum $\square$ stark $\quad$ keine Angabe

Wiederaufbau
Instandsetzung
Einsatzmassnahmen
Einsatzvorbereitung
Vorsorge
Prävention (techn. Massnahmen)
Prävention (plan. Massnahmen)

$13 \%$

$15 \%$

$30 \%$

$36 \%$ $21 \%$

\begin{tabular}{|c|c|c|c|}
\hline $11 \%$ & $36 \%$ & $34 \%$ & $19 \%$ \\
\hline $9 \%$ & $42 \%$ & $26 \%$ & $23 \%$ \\
\hline
\end{tabular}

\begin{tabular}{|l|l|l|l|}
\hline $9 \%$ & $42 \%$ & $26 \%$ & $23 \%$ \\
\hline $11 \%$ & $32 \%$ & $38 \%$ & $19 \%$ \\
\hline
\end{tabular}

Abb. 5 Relevanz des Bevölkerungsrückgangs in Bezug auf den Risikokreislauf

ist (Abb. 5). Im Zusammenhang mit der sich verändernden Bevölkerungsstruktur hinsichtlich Größe und Altersstruktur, aber auch Aufenthaltsdauer der Personen in den (potenziell) betroffenen Gemeinden bzw. Orten, stellt sich zudem die Frage, wie mit dem Thema Nebenwohnsitze bzw. der Personengruppe von NebenwohnsitznehmerInnen bzw. multilokal Lebenden im Zusammenhang mit dem lokalen Hochwassermanagement adäquat umgegangen werden kann. Dies ist deshalb von Bedeutung, weil VertreterInnen dieser Gruppe in unterschiedlichem Ausmaß und in unterschiedlicher Periodizität vor Ort sind und sich in der Regel - vielleicht auch deshalb - häufiger nicht auf ein Ereignis vorbereiten. Darüber hinaus ist anzunehmen, dass diese Personengruppe im Einsatzfall tendenziell seltener als (aktive) Mitglieder freiwilliger Blaulichtorganisationen zur Verfügung steht.

Darüber hinaus sehen die meisten Befragten im Bereich der Instandhaltung von technischen Maßnahmen eine nicht unwesentliche Verbindung zu demografischen Entwicklungen. Da die Gemeinden für deren Finanzierung aufkommen müssen, können rückläufige Bevölkerungszahlen und die daraus resultierende Reduktion der Steuereinnahmen zu Schwierigkeiten führen.

\section{Fazit und Ausblick}

Der demografische Wandel ist das komplexe Ergebnis des Zusammenwirkens von quantitativen und altersstrukturellen Veränderungen im Aufbau der
Bevölkerung, einer zunehmenden Heterogenität der Bevölkerung (auch auf kleinsträumiger Ebene) in Bezug auf die Wanderungsbiographien sowie Entwicklungen des sozioökonomischen Status und geänderten Lebensstilen, wie z.B. Pendeln, Einpersonenhaushalte und Mehrgenerationenhaushalte. Dies verlangt nach einer multidimensionalen Sichtweise nicht nur auf Seiten der Wissenschaft, sondern auch auf Seiten der Politik und Fachbehörden. Da sich der demografische Wandel langsam und daher oft unbemerkt vollzieht, werden dessen Konsequenzen oft ignoriert bzw. lassen sich über lange Zeit hinweg verdrängen. Dies gelingt bis $\mathrm{zu}$ jenem Moment, in dem Extremereignisse auf „problematische“ demografische (örtliche) Gegebenheiten treffen und sich schlagartig sämtliche tangierte bzw. involvierte Akteure mit großen operativen Herausforderungen konfrontiert sehen. Die größten Herausforderungen in Bezug auf die Vorsorge werden in der zunehmenden sozialen Verwundbarkeit der Bevölkerung in alpinen ländlichen Räumen gesehen. Hier wird der Anteil an älteren und damit von externer professioneller Betreuung und Pflege abhängigen Ereignisfall bzw. in der Phase des Wiederaufbaus periphere, dünn besiedelte Regionen vor zentrale Herausforderungen stellen wird. Gleichzeitig kann der demografische Wandel auch als Ansatzpunkt für die Veränderung des Hochwasserrisikos und dessen Management dienen (Fuchs et al. 2015, 2017; Rogger et al. 2017). Im Bereich des HochwasserMenschen zunehmen, was vor allem im risikomanagements ist der Einfluss des demografischen Wandels differenzierter zu betrachten, da sich dieser Prozess in unterschiedlichem Ausmaß auf alle Phasen des Risikokreislaufs auswirkt. Die Ergebnisse der Umfrage legen nahe, die Konsequenzen des demografischen Wandels vor allem für die Rekrutierung und Bereitstellung einer ausreichenden Anzahl an Personal für den Einsatzfall und damit für die Gewährleistung der Erfüllung der notwendigen Aufgaben ernst zu nehmen. Dazu kann auch ein größeres Engagement in politischen und öffentlichen Diskussionen beitragen. In weiterer Folge müssten sich die gesteigerte Aufmerksamkeit und das Bewusstsein für das Thema in relevanten politischen Entscheidungsprozessen widerspiegeln. Um im Ereignisfall gut vorbereitet zu sein, muss es auf der Verwaltungsebene gelingen, in einem ersten Schritt alle relevanten Fachabteilungen - allen voran das Katastrophenmanagement, die Raumplanung, die Schutzwasserwirtschaft sowie die Wildbach- und Lawinenverbauung zusammenzuführen, um im Kontext des Risikomanagements explizit versorgungs- und schutzbezogene Aufgaben im Lichte der Konsequenzen des demografischen Wandels zu diskutieren. Eine breite Diskussion unter Mitwirkung aller relevanten Akteure ist deshalb so wichtig, weil die daraus resultierenden Entscheidungen von langfristiger Tragweite sind. Das Fehlen einer diesbezüglichen antizipativen Vorgangsweise weist aktuell Parallelen zur Berücksichtigung des Klimawandels im Hochwasserrisikomanagement auf (Nordbeck et al. 2019). Ein derartiger Prozess wird allerdings dadurch erschwert, dass sich die Aufgaben und Kompetenzen der jeweiligen Fachabteilungen auf verschiedene Themenbereiche beziehen. Auch diese institutionelle Hürde muss übersprungen werden, um die Komplexität des demografischen Wandels adäquat berücksichtigen zu können.

Um dem demografischen Wandel im Hochwasserrisikomanagement zukünftig entsprechend Rechnung zu tragen, bedarf es neben einer verstärkten integrativ-strategischen Vorgangsweise auf Ebene des Bundes und der Länder eines Reflektierens der Logiken und langfristigen Eignung der bisherigen Anpassungsstrategien sowie Mut zu neuen raumangepassten Herangehensweisen. In diesem Zusammenhang bietet sich die Intensivierung des interund transdisziplinären Dialogs an. 
Förderung Wir danken der Österreichischen Akademie der Wissenschaften (ÖAW) für die Förderung des Projekts „DemoHazAlps (Demographic change and hydrological hazards: flood risk management in Alpine areas facing population decline and demographic ageing)“, in dessen Rahmen der vorliegende Beitrag erarbeitet wurde.

Funding Open access funding provided by University of Natural Resources and Life Sciences Vienna (BOKU).

Open Access Dieser Artikel wird unter der Creative Commons Namensnen- nung 4.0 International Lizenz veröffentlicht, welche die Nutzung, Vervielfältigung, Bearbeitung, Verbreitung und Wiedergabe in jeglichem Medium und Format erlaubt, sofern Sie den/die ursprünglichen Autor(en) und die Quelle ordnungsgemäß nennen, einen Link zur Creative Commons Lizenz beifügen und angeben, ob Änderungen vorgenommen wurden.

Die in diesem Artikel enthaltenen Bilder und sonstiges Drittmaterial unterliegen ebenfalls der genannten Creative Commons Lizenz, sofern sich aus der Abbildungslegende nichts anderes ergibt. Sofern das betreffende Material nicht unter der genannten Creative Commons Lizenz steht und die betreffende Handlung nicht nach gesetzlichen Vorschriften erlaubt ist, ist für die oben aufgeführten Weiterverwendungen des Materials die Einwilligung des jeweiligen Rechteinhabers einzuholen.

Weitere Details zur Lizenz entnehmen Sie bitte der Lizenzinformation auf http://creativecommons.org/licenses/ by/4.0/deed.de.

\section{Literatur}

Balas, M., Glas, N., Seebauer, S., Liehr, C., Pfurtscheller, C., Fordinal, I., Babcicky, P. (2015): Freiwilligenengagement in der Zukunft. Maß nahmen für die langfristige Absicherung der Freiwilligenarbeit im Katastrophenschutz. Umweltbundesamt, Wien

Bätzing, W. (2015): Die Alpen. Geschichte und Zukunft einer europäischen Kulturlandschaft, 4. Auflage, Beck, München

Birkmann, J., Cutter, S.L., Rothman, D.S., Welle, T., Garschagen, M., van Ruijven, B., O'Neill, B., Preston, B.L., Kienberger, S., Cardona, O.D. Siagian, T., Hidayati, D., Setiadi, N., Binder, C.R., Hughes, B., Pulwarty, R. (2015): Scenarios for vulnerability: Opportunities and constraints in the context of climate change and disaster risk. Climatic Change 133 (1): 53-68

BMASK (2015): 2. Freiwilligenbericht 2015. Bericht zur Lage und zu den Perspektiven des freiwilligen Engagements in Österreich. Sozialministerium, Wien

Brown, D. L. (2010): Rethinking the OECD's new rural demography. Centre for Rural Economy Discussion Paper Series No. 26, Newcastle.

Bundesamt für Bevölkerungsschutz (BABS) (2014): Integrales Risikomanagement: Bedeutung für den Schutz der Bevölkerung und ihrer Lebensgrundlagen. Bern.

Báchora, R., Leitner, K., Steinheimer, E. (2016): Herausforderungen des demographischen Wandels - Strategien und Konzepte der Politik und Wirtschaft. Österreich im Ländervergleich mit Deutschland, Großbritannien und Schweden. Wien: Center für Strategische Analysen.

Clar, C. (2019): How demographic developments determine the management of hydrometeorological hazard risks in rural communities: The linkages between demographic and natural hazards research. WIREs Water 6 (6): el378

CoR (Ed.) (2016): The impact of demographic change on European regions. Brussels: European Union-Commitee of the Regions

Cutter, S.L., Boruff, B.J., Shirley, W.L. (2003): Social Vulnerability to Environmental Hazards* Social Science Quarterly 84 (2):242-261

Fekete, A. (2009a): The Interrelation of Social Vulnerability and Demographic Change in Germany. Paper at the IHDP Open Meeting 2009, the 7th International Science Conference on the Human Dimensions of Global Environmental Change.

Fekete, A. (2009b): Validation of a social vulnerability index in context to river-floods in Germany. Natural Hazards and Earth System Sciences 9 (2):393-403

Fekete, A. (2018): Social vulnerability change assessment: monitoring longitudinal demographic indicators of disaster risk in Germany from 2005to 2015. Natural Hazards 95 (3):585-614.

Fuchs, S, Keiler M, Zischg A (2015) A spatiotemporal multi-hazard exposure assessment based on property data. Natural Hazards and Earth System Sciences 15:2127-2142

Fuchs, S., Röthlisberger, V., Thaler, T., Zischg, A., Keiler, M. (2017): Natural hazard management from a co-evolutionary perspective: exposure and policy response in the European Alps. Annals of the American Association of Geographers 107:382-392

Karagiorgos, K., Thaler, T., Heiser, M., Hübl, J., Fuchs, S. (2016): Integrated flash flood vulnerability assessment: insights from East Attica, Greece. Journal of Hydrology 541 (Part A):553-562
Nordbeck, R., Steurer, R., Löschner, L. (2019): The future orientation of Austria's flood policies: from flood control to anticipatory flood risk management. Journal of Environmental Planning and Management 62 (11):1864-1885

Österreichische Raumordnungskonferenz [ÖROK] (Hrsg.) (2015): ÖROK-Regionalprognosen 2014-2030. Teil 1: Bevölkerung

Rogger, M., Agnoletti, M., Alaoui, A., Bathurst, J.C., Bodner, G., Borga, M., Chaplot, V., Gallart, F, Glatzel, G., Hall, J., Holden, J., Holko, L., Horn, R., Kiss, A., Kohnova, S., Leitinger G., Lennartz, B., Parajka, J., Perdigao, R., Peth, S., Plavcova, L., Quinton, J.N., Robinson, M., Salinas, J.L., Santoro, A., Szolgay, J., Tron, S., van den Akker, J.J.H., Viglione, A., Blöschl, G. (2017): Land use change impacts on floods at the catchment scale: Challenges and opportunities for future research. Earth and Space Science: 5209-5219

Statistik Austria (2017): Demographisches Jahrbuch 2016. Bundesanstalt Statistik Austria, Wien. Szigetvari A (2018): Warum der Westen für Arbeitssuchende oft wenig reizvoll ist. DerStandard, 19.09.2018. https://www.derstandard.at/ story/2000087602726/warum-der-westen-fuerarbeitssuchende-oft-wenig-reizvoll-ist

Tappeiner U, Borsdorf A, Tasser E (eds.) (2008) Atlas Alps. Mapping the Alps. Society-EconomyEnvironment. Spektrum Akademischer Verlag, Heidelberg

Hinweis des Verlags Der Verlag bleibt in Hinblick auf geografische Zuordnungen und Gebietsbezeichnungen in veröffentlichten Karten und Institutsadressen neutral. 